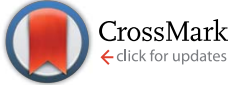

Cite this: J. Mater. Chem. A, 2016, 4, 9014

Received 16th April 2016 Accepted 17th May 2016

DOI: $10.1039 / c 6 t a 03158 g$

www.rsc.org/MaterialsA

\section{Highly stable hollow bifunctional cobalt sulfides for flexible supercapacitors and hydrogen evolution $\dagger$}

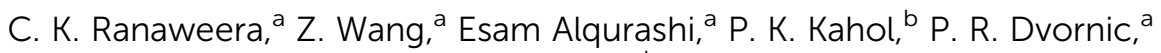 \\ Bipin Kumar Gupta, ${ }^{c}$ Karthik Ramasamy, ${ }^{d}$ Aditya D. Mohite, ${ }^{e}$ Gautam Gupta ${ }^{e}$ \\ and Ram K. Gupta*a
}

Hollow structures of NiAs-type cobalt sulfide have been synthesized by a facile hydrothermal method. These hollow structured cobalt sulfides exhibit excellent electrochemical properties for supercapacitor applications $\left(867 \mathrm{~F} \mathrm{~g}^{-1}\right.$ ) and respectable hydrogen evolution activity. The symmetrical supercapacitor device fabricated using cobalt sulfide nanostructures showed an areal capacitance of $260 \mathrm{mF} \mathrm{cm}{ }^{-2}$ with good flexibility and high temperature stability. The specific capacitance of the supercapacitor is enhanced over $150 \%$, when the temperature is increased from 10 to $70^{\circ} \mathrm{C}$.

Over the last few decades, the increasing demand for energy has motivated the scientific community to develop high performance, sustainable, cost-effective and environmentally friendly materials for energy conversion and storage applications. ${ }^{1-4}$ Undoubtedly, materials for supercapacitors and hydrogen evolution are among the widely studied ones for these purposes. Supercapacitors are electrochemical energy storage devices primarily appealing for their excellent power density and long cycle life. ${ }^{4-7}$ Carbon based electrodes are suitable for delivering high power density but have low energy density. ${ }^{8}$ Nevertheless, in recent years, the energy density of supercapacitors has been boosted by the use of metal oxides, which in turn sacrifices the cycle life. $^{4}$

Second, energy generation using a cleaner and cheaper method is of high importance due to increasing concerns of carbon emission. Hydrogen generation by splitting water is

${ }^{a}$ Department of Chemistry, Pittsburg State University, 1701 S. Broadway, Pittsburg, KS 66762, USA.E-mail: ramguptamsu@gmail.com

${ }^{b}$ Department of Physics, Pittsburg State University, 1701 S. Broadway, Pittsburg, KS 66762, USA

'National Physical Laboratory (CSIR), Dr K. S. Krishnan Road, New Delhi 110012, India

${ }^{d}$ Center for Integrated Nanotechnologies, Los Alamos National Laboratory, Albuquerque, NM-87185, USA

${ }^{e}$ Materials Physics and Application Division, Los Alamos National Laboratory, Los Alamos, NM-87545, USA

$\dagger$ Electronic supplementary information (ESI) available: Synthesis, characterization and some additional figures. See DOI: 10.1039/c6ta03158g highly desired. The hydrogen evolution reaction (HER) is one of the key steps in the water splitting process. ${ }^{9}$ Ideally, the thermodynamic potential for the hydrogen evolution reaction should be at $0 \mathrm{~V}$ (vs. SHE). However, without an efficient catalyst, this reaction occurs at high overpotential. ${ }^{10}$ Presently, platinum is the most effective and durable catalyst for the hydrogen evolution reaction, but its widespread use is precluded due to its high cost and limited availability.

At this juncture, it is essential to develop cost effective, sustainable and stable materials for both supercapacitor and HER applications. Recently, transition metal chalcogenides have attracted considerable attention for energy applications such as fuel cells, supercapacitors and batteries due to their high abundance, low cost and decent performance., ${ }^{2,11-15}$ Wang et al. have synthesized 3D flower-like $\mathrm{MoS}_{2}$ nanostructures and used them as an electrode for high-performance electrochemical capacitors. ${ }^{16}$ The $\mathrm{MoS}_{2}$ electrode showed a specific capacitance of $168 \mathrm{~F} \mathrm{~g}^{-1}$ and retained $92.6 \%$ of capacitance even after 3000 cycles. Soon et al. have reported a $\mathrm{MoS}_{2}$ based supercapacitor having a capacitance of $8 \mathrm{mF} \mathrm{cm}{ }^{-2} \cdot{ }^{17}$ Hierarchically porous nickel sulfide has been synthesized for both the HER and oxygen evolution reaction (OER). ${ }^{18}$ The synthesized nickel sulfide showed overpotentials of $60 \mathrm{mV}$ for the HER and $180 \mathrm{mV}$ for the OER in $1.0 \mathrm{M} \mathrm{KOH}$ at a current density of $10 \mathrm{~mA} \mathrm{~cm}{ }^{-2}$, respectively. Sharma et al. have synthesised flower-like $\mathrm{ZnS}$ for charge storage applications. ${ }^{19}$ They observed a high specific capacitance $\left(226 \mathrm{~F} \mathrm{~g}^{-1}\right)$ and specific capacitance was observed to decrease by $60 \%$ on increasing the scan rate from 20 to $200 \mathrm{mV} \mathrm{s}^{-1}$. Other metal sulfides such as $\mathrm{WS}_{2}$ are also synthesized for HER, supercapacitor and battery applications..$^{20-22}$ As an important member of the transition metal sulfide family, cobalt sulfide is considered to be one of the promising candidates for such applications. Accordingly, various phases of cobalt sulfides have been synthesized and studied for supercapacitor and HER applications..$^{23-29}$

Xing et al. have synthesized octahedron-shaped $\mathrm{CoS}_{2}$ crystals using a hydrothermal method for their application in supercapacitors. ${ }^{30}$ These $\mathrm{CoS}_{2}$ crystals showed a specific capacitance 
of $237 \mathrm{~F} \mathrm{~g}^{-1}$ at $1 \mathrm{~A} \mathrm{~g}^{-1}$ with only a loss of $7.4 \%$ in the specific capacitance after 2000 cycles. Sphere-like CoS nanostructures were synthesized and used as an electrode material for charge storage applications. ${ }^{31}$ These sphere-like CoS nanostructures showed very stable electrochemical performance with only about $13 \%$ reduction in the charge storage capacity of the available capacitance $\left(363 \mathrm{~F} \mathrm{~g}^{-1}\right.$ ) on increasing the scan rate by 10 times. Ray et al. have used a facile electrochemical technique to fabricate titania nanotube-CoS composite electrodes for supercapacitor applications. ${ }^{32}$ Cyclic voltammetric measurements presented a very high specific capacitance $\left(370 \mathrm{~F} \mathrm{~g}^{-1}\right)$ with great cycle stability in $\mathrm{KOH}$ electrolytes. Since various phases of cobalt sulfides have been found useful for energy storage and catalytic applications, it is highly desirable to synthesize cobalt sulfide using a facile and efficient method to explore its multifunctionality. Herein, we report a facile one step hydrothermal synthesis of hollow structured NiAs-type cobalt sulfide and investigate the material for supercapacitor and HER applications. Our investigations show that cobalt sulfide exhibits excellent electrochemical properties for supercapacitor applications with a specific capacitance of $867 \mathrm{~F} \mathrm{~g}^{-1}$ and can be used as an electrocatalyst for the hydrogen evolution reaction (Tafel slope of $97 \mathrm{mV}$ per decade). The high performance of the cobalt sulfide could be due to its unique morphology.

Hollow structured cobalt sulfide was synthesized by reacting cobalt nitrate with thioacetamide in ethylene glycol under hydrothermal conditions at $180{ }^{\circ} \mathrm{C}$ for $12 \mathrm{~h}$ (details in the ESI $\dagger$ ). The powder XRD pattern of the black precipitate from the hydrothermal reaction showed diffraction planes, predominantly matching with the NiAs-type hexagonal $\mathrm{Co}_{1-x} \mathrm{~S}$ phase of cobalt sulfide (JCPDS file: 42-0826). The diffraction peaks can be indexed to the (010), (011), (012), and (120) planes of $\mathrm{Co}_{1-x} \mathrm{~S}$
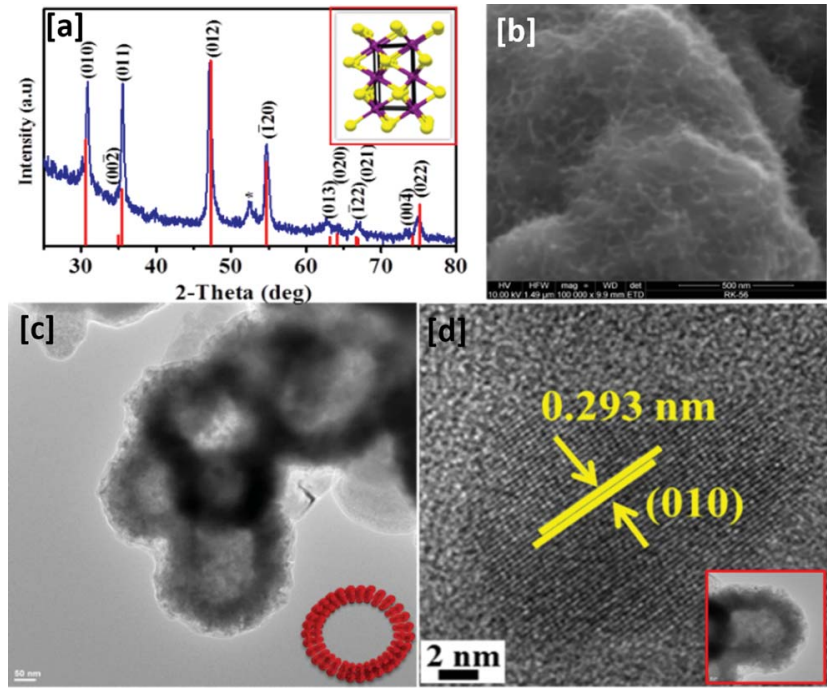

Fig. 1 (a) XRD pattern (inset: crystal structure of $\mathrm{CO}_{1-x} \mathrm{~S}$ ), (b) SEM image, (c) TEM image (inset: schematic of the hollow structure composed of nanocrystals), and (d) HRTEM image of synthesized cobalt sulfide (inset: low magnification TEM image of the hollow structure).
(Fig. 1a). The scanning electron microscopy image of the cobalt sulfide obtained by a hydrothermal reaction showed irregularly shaped granules (Fig. 1b). The ratio of cobalt to sulfur determined from SEM-EDX was found to be 0.98, close to the expected composition for $\mathrm{Co}_{1-x} \mathrm{~S}$ (Fig. 1S, ESI $\dagger$ ). To gain morphological insight, TEM imaging was performed. The TEM image in Fig. 1c shows nearly uniform hollow ring-like structures that are composed of pseudo-spherical nanocrystals. It is widely accepted that the hollow structures are a consequence of the difference in diffusion rates of constituent elements. ${ }^{33}$ A similar hollow structure has also been reported by microwave assisted synthesis of cobalt sulfide. ${ }^{33}$ The lattice distance measured from the HRTEM image (Fig. 1d) of $\mathrm{Co}_{1-x} \mathrm{~S}$ nanocrystals was close to $0.293 \mathrm{~nm}$ corresponding to the (010) plane. In addition, we have carried out BET nitrogen adsorption/desorption isotherm measurements to determine the surface area of the sample (Fig. 2S, ESI $\dagger$ ). The hysteresis loop in the isotherm of the synthesized $\mathrm{Co}_{1-x} \mathrm{~S}$ is an indication of capillary condensation, which arises due to different mechanisms of adsorption/desorption in micropores. The synthesized $\mathrm{Co}_{1-x} \mathrm{~S}$ showed a specific surface area of $23.9 \mathrm{~m}^{2} \mathrm{~g}^{-1}$ which is much higher than that of commercial samples of $\operatorname{CoS}\left(0.39 \mathrm{~m}^{2} \mathrm{~g}^{-1}\right)$ and $\mathrm{MoS}_{2}\left(3.33 \mathrm{~m}^{2} \mathrm{~g}^{-1}\right)$.

The electrochemical properties of the electrode prepared using $\mathrm{Co}_{1-x} \mathrm{~S}$ hollow structures were studied using cyclic voltammetry (CV), galvanostatic charge-discharge (GCD), and electrochemical impedance spectroscopy (EIS). The CV measurements of the $\mathrm{Co}_{1-x} \mathrm{~S}$ electrode in an alkaline electrolyte (3 $\mathrm{M} \mathrm{NaOH}$ ) displayed non-rectangular shaped curves with quasi-reversible redox waves in a potential range of 0 to $0.6 \mathrm{~V}$ (Fig. 2a). The unambiguous appearance of redox waves confirms the pseudocapacitive behaviour of $\mathrm{Co}_{1-x} \mathrm{~S}$.

As evident, the charge-storage mechanism of CoS is significantly faradic in nature rather than capacitive. The faradic
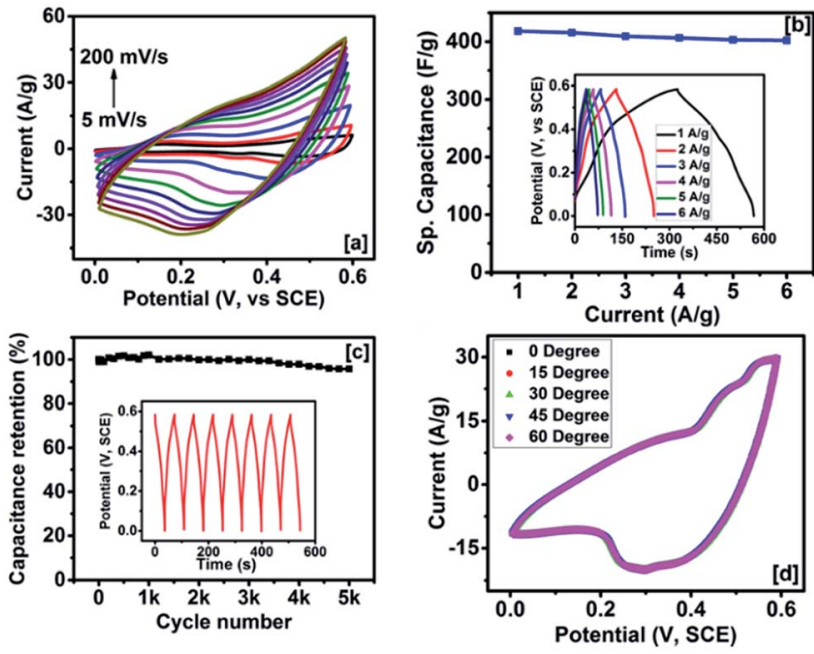

Fig. 2 (a) CV curves at various scan rates, (b) variation of specific capacitance with applied current (inset figure shows potential-time curves at different applied currents), (c) capacitance retention as a function of number of charge-discharge cycles (inset figure shows the first few cycles of charge-discharge curves), and (d) CV curves at various bending angles of the cobalt sulfide electrode. 
reaction occurring on the surface of the CoS electrode in alkaline media is given below: ${ }^{31}$

$$
\begin{gathered}
\mathrm{CoS}+\mathrm{OH}^{-} \leftrightarrow \mathrm{CoSOH}+\mathrm{e}^{-} \\
\mathrm{CoSOH}+\mathrm{OH}^{-} \leftrightarrow \mathrm{CoSO}+\mathrm{H}_{2} \mathrm{O}+\mathrm{e}^{-}
\end{gathered}
$$

The specific capacitance of the $\mathrm{Co}_{1-x} \mathrm{~S}$ electrode estimated from $\mathrm{CV}$ curves was found to be $867 \mathrm{~F} \mathrm{~g}^{-1}$ at $5 \mathrm{mV} \mathrm{s}^{-1}$ (Fig. 3S, ESI $\dagger$ ). Further, GCD measurements exhibit symmetrical and non-linear charge-discharge characteristics, confirming the pseudocapacitance behaviour of the electrode (inset of Fig. 2b). The specific capacitance of the cobalt sulfide electrode determined from GCD measurements is about $420 \mathrm{~F} \mathrm{~g}^{-1}$ at $1 \mathrm{~A} \mathrm{~g}^{-1}$, which is significantly higher than the value obtained for CoS hollow nanosheets and hollow nanoprisms. ${ }^{31,33}$ Interestingly, the specific capacitance value was nearly retained $\left(402 \mathrm{~F} \mathrm{~g}^{-1}\right.$ at $6 \mathrm{~A} \mathrm{~g}^{-1}$ ) even at higher current density (Fig. 2b), suggesting excellent rate stability of $\mathrm{Co}_{1-x} \mathrm{~S}$ hollow structures. In addition, we have evaluated the cyclic stability of the cobalt sulfide electrode using GCD measurements. From Fig. 2c, it can be witnessed that the specific capacitance was almost retained even after 5000 charge-discharge cycles, confirming its high electrochemical stability. It is noteworthy that the electrode using $\mathrm{CoS}_{2}$ ellipsoids lost nearly $50 \%$ of specific capacitance within 1000 charge-discharge cycles. ${ }^{34}$ Furthermore, we have tested the flexibility of the cobalt sulfide electrodes by carrying out CV measurements at different bending angles. The shape and size of the $\mathrm{CV}$ curves were found to be identical at different bending angles (Fig. 2d), suggesting that $\mathrm{Co}_{1-x} \mathrm{~S}$ hollow structures are exceptional candidates for flexible energy storage devices. The variation of the specific energy density versus power density (Ragone plot) for cobalt sulfide is shown in Fig. 4S (ESI $\dagger$ ). The highest specific energy density of $19.4 \mathrm{~W} \mathrm{~h} \mathrm{~kg}^{-1}$ and power density of $2644 \mathrm{~W} \mathrm{~kg}^{-1}$ were observed.

We fabricated the supercapacitor device by sandwiching an ion transporting layer between two cobalt sulfide electrodes (inset of Fig. 3b). Fig. 3a shows the CV curves of the supercapacitor device at different scan rates. The shape of the voltammogram at various scan rates is similar in nature, suggesting high charge transfer stability of the device even at high scan rates. The nearly rectangular shape of the CV curves indicates a near ideal capacitive behaviour. The variation of specific capacitance as a function of scan rate for the device is shown in Fig. 3b. The specific capacitance of the device was observed to decrease with the increase in the scan rate. The highest areal capacitance of $260 \mathrm{mF} \mathrm{cm}{ }^{-2}$ was observed at a scan rate of $5 \mathrm{mV} \mathrm{s}^{-1}$. It was observed that specific capacitance of the devices decreases with increasing scan rate, which is due to insufficient time for the electrolyte to form an electrochemical double layer capacitor at the cobalt sulfide electrode. The galvanostatic charge-discharge measurements were also performed to study the charge storage capacity of the device. The charge storage capacity of the device decreases with increasing current density (Fig. 5S, ESI $\dagger$ ). A specific capacitance of $60 \mathrm{mF} \mathrm{cm} \mathrm{cm}^{-2}$ was observed at $2 \mathrm{~mA} \mathrm{~cm}{ }^{-2}$ current density. Further, the effect of temperature on the performance of the
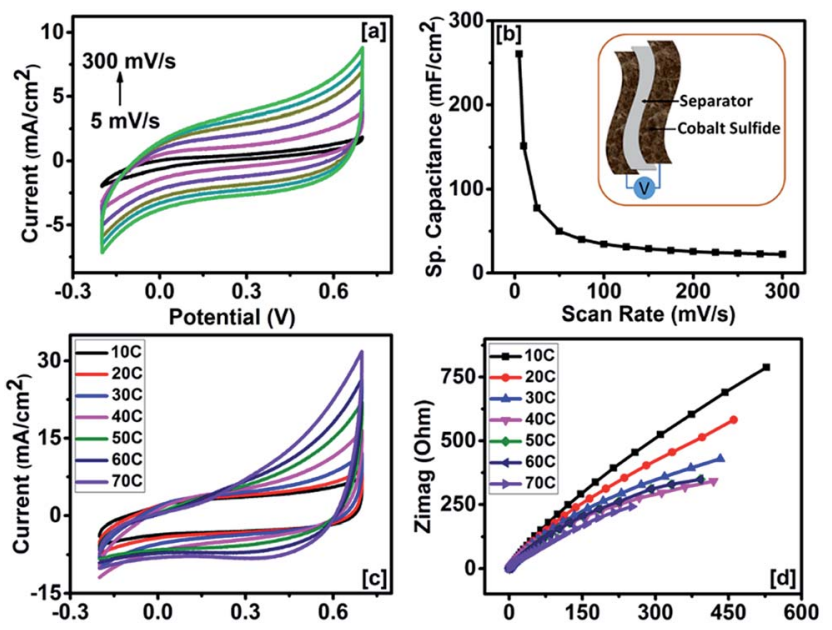

Fig. 3 (a) CV curves of the supercapacitor device at various scan rates at room temperature, (b) variation of specific capacitance as a function of scan rate (inset figure shows the schematic diagram of the supercapacitor device), (c) CV curves of the supercapacitor device at different temperatures, and (d) $Z_{\text {real }} v s$. $Z_{\text {imag }}$ plots of the device at different temperatures.

supercapacitor device was studied. The CV curves of the device at various temperatures are shown in Fig. 3c. The area under the $\mathrm{CV}$ curves increases with increasing temperature, indicating improvement in the charge storage capacity of the device. It was observed that the charge storage capacity of the device increases over $150 \%$ by increasing temperature from $10{ }^{\circ} \mathrm{C}$ to $70{ }^{\circ} \mathrm{C}$ (Fig. 6S, ESI $\dagger$ ). The improved performance of the cobalt sulfide based supercapacitor device could be due to activation of the cobalt sulfide sites at higher temperatures. In addition, the improvement could be due to improved mobility of the electrolyte ions and lower series resistance at higher temperatures.

The effect of temperature on the electrochemical behaviour of the supercapacitor was further investigated using electrochemical impedance spectroscopy (EIS). The variation of real and imaginary impedance of the supercapacitor device at various temperatures is shown in Fig. 3d. The real and imaginary impedance of the device decreases with increasing temperature. The equivalent series resistance (ESR) of the supercapacitor device decreases with increasing temperature (Fig. 7S, ESI $\dagger$ ). The decrease in ESR with temperature could affect the charge storage properties of the device. The increase in the temperature enhances the mobility of the electrolyte ions and thus increases the conductivity of the electrolyte ions. ${ }^{35}$ The effect of temperature on the total impedance of the device is shown in Fig. 8S, ESI. $\dagger$ It was observed that the impedance of the cobalt sulfide based supercapacitor device decreases with increasing temperature. The total impedance of the supercapacitor device was higher at lower frequency compared to that of at higher frequency. Our observations based on electrochemical characterization suggest that the cobalt sulfide based supercapacitor device performs even better at elevated temperatures than at room temperature.

The HER activity of the synthesized cobalt sulfide was also studied using a glassy carbon working electrode with a standard 
rotating disk electrode instrument. All measurements were performed in $1 \mathrm{~N} \mathrm{H}_{2} \mathrm{SO}_{4}$ (aq) electrolyte under continuous purging with nitrogen gas (details in the ESI $\dagger$ ). We have compared the HER activity of commercially available molybdenum sulfide $\left(\mathrm{MoS}_{2}\right.$-CS, Sigma, USA) and cobalt sulfide (CoS-CS, Strem Chemicals, USA) with that of our cobalt sulfide hollow structures. The polarization curves of the samples are shown in Fig. 4a. Cobalt sulfide showed a lower onset potential (0.21 V vs. RHE) compared to $\mathrm{MoS}_{2}$-CS (0.31 V vs. RHE) and CoS-CS (0.35 V vs. RHE). Our synthesized cobalt sulfide displayed about 60 and 30 times higher current density at $0.30 \mathrm{~V}$ (vs. RHE) compared with $\mathrm{MoS}_{2}$-CS and CoS-CS, respectively. The HER activity was further analysed using corresponding Tafel plots (inset of Fig. 4a). A Tafel slope of $97 \mathrm{mV}$ per decade, $174 \mathrm{mV}$ per decade and $113 \mathrm{mV}$ per decade was observed for synthesized cobalt sulfide, $\mathrm{MoS}_{2}$-CS and CoS-CS, respectively. Lukowski et al. have reported a Tafel slope of $112 \mathrm{mV}$ per decade for as such synthesized nanostructured $\mathrm{MoS}_{2} \cdot{ }^{36} \mathrm{~A}$ Tafel slope of $75 \mathrm{mV}$ per decade and $90 \mathrm{mV}$ per decade was reported for CoS synthesized using microwave assisted and solvothermal methods, respectively. ${ }^{33}$ Sun et al. have used electrodeposited cobalt-sulfide for HER applications and reported a Tafel slope of $93 \mathrm{mV} \mathrm{s}^{-1}$ in phosphate buffer solution. ${ }^{26} \mathrm{CoS}_{2}$ synthesized using a hydrothermal method showed a Tafel slope of $72 \mathrm{mV} \mathrm{s}^{-1}$ in acidic media. ${ }^{37}$ Peng et al. have synthesized $\mathrm{CoS}_{2} / \mathrm{RGO} / \mathrm{CNT}$ nanocomposites for the HER. ${ }^{38}$ A Tafel slope of about 51, 82 and $148 \mathrm{mV} \mathrm{s}^{-1}$ was observed for $\mathrm{CoS}_{2} / \mathrm{RGO} / \mathrm{CNT}, \mathrm{CoS}_{2} / \mathrm{RGO}$ and $\mathrm{CoS}_{2}$, respectively. The rate of hydrogen evolution is limited either by proton adsorption onto an active site or evolution of the formed hydrogen from the surface. A high Tafel slope (120 $\mathrm{mV}$ per decade) is indicative of proton adsorption (Volmer step) as the rate-limiting step, while a lower Tafel slope (30 or $40 \mathrm{mV}$ per decade) indicates that the evolution of molecular hydrogen from the catalyst is rate limiting (Heyrovsky or Tafel step, respectively). A Tafel slope of $97 \mathrm{mV}$ per decade for $\mathrm{Co}_{1-x} \mathrm{~S}$ suggests the typical Volmer-Tafel route with the Volmer step as the rate determining step. ${ }^{39}$ Electrochemical impedance spectra of the cobalt sulfide were recorded at various potentials (Fig. 4b). As seen in the EIS spectra, the arc at zero potential converges to a semi-circle at negative potentials. A decrease in the semi-circle diameter is observed with the increase in negative potential. The charge-transfer resistance determined from the semicircle registered at low frequencies was found to
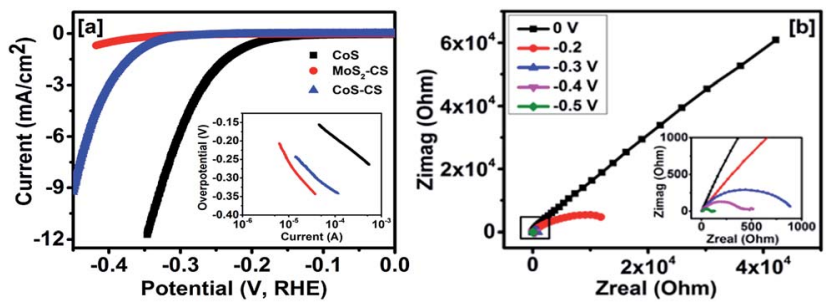

Fig. 4 (a) Polarization curves of synthesized cobalt sulfide (CoS) and commercial samples of molybdenum sulfide $\left(\mathrm{MoS}_{2}-\mathrm{CS}\right)$ and cobalt sulfide (COS-CS) (inset figure shows the Tafel plots) and (b) $Z_{\text {real }} v s$. $Z_{\text {imag }}$ plots at different potentials for cobalt sulfide. be overpotential-dependent. A similar observation was reported for molybdenum phosphide. ${ }^{9}$

In conclusion, we have synthesized nanostructured cobalt sulfide by a facile hydrothermal method. The charge storage capacity and hydrogen evolution reaction activity were investigated using electrochemical methods. The highest specific capacitance of $867 \mathrm{~F} \mathrm{~g}^{-1}$ was observed at a scan rate of $5 \mathrm{mV} \mathrm{s}^{-1}$ in cyclic voltammetry measurements. Cobalt sulfide showed a very stable electrochemical behaviour up to 5000 cycles of charge-discharge study with highly flexible nature. The symmetrical supercapacitor device showed an areal capacitance of about $260 \mathrm{mF} \mathrm{cm} \mathrm{cm}^{-2}$ which was improved over $150 \%$ on increasing the device temperature from 10 to $70{ }^{\circ} \mathrm{C}$. The HER activity of cobalt sulfide was observed to be better than that of commercially available molybdenum sulfide. Our study suggests that cobalt sulfide could be used for high temperature operable flexible energy storage devices and as an electrocatalyst for the hydrogen evolution reaction.

\section{Author contributions}

RKG designed the experiments, ran some structural and electrochemical characterization of the devices, interpreted and analyzed the data, and prepared the manuscript. CR, CZ, and EA helped in synthesis and performed some electrochemical characterization. KR performed TEM measurements. GG and AM guided the hydrogen evolution studies. PK, PD, KR, BKG and GG reviewed the manuscript. All the authors reviewed and commented on the manuscript.

\section{Conflict of interests}

The authors declare no competing financial interests. Requests for materials should be addressed to RKG.

\section{Acknowledgements}

Dr Ram K. Gupta expresses his sincere acknowledgment to the Polymer Chemistry Initiative, Pittsburg State University for providing financial and research support. This material is partly based upon work supported by the National Science Foundation under Award No. EPS-0903806 and matching support from the State of Kansas through the Kansas Board of Regents. Dr Ram K. Gupta acknowledges support from the Department of Energy for Visiting Faculty Program. The authors thank Dr Lifeng Dong for recording SEM/EDX. Dr G. Gupta and A. Mohite acknowledge Los Alamos LDRD.

\section{References}

1 M. Winter and R. J. Brodd, Chem. Rev., 2004, 104, 4245-4270. 2 K. Ramasamy, R. K. Gupta, S. Palchoudhury, S. Ivanov and A. Gupta, Chem. Mater., 2015, 27, 379-386.

3 K. Ramasamy, H. Sims, W. H. Butler and A. Gupta, J. Am. Chem. Soc., 2014, 136, 1587-1598.

4 G. Wang, L. Zhang and J. Zhang, Chem. Soc. Rev., 2012, 41, 797-828. 
5 B. E. Conway, V. Birss and J. Wojtowicz, J. Power Sources, 1997, 66, 1-14.

6 F. Zhao, Y. Wang, X. Xu, Y. Liu, R. Song, G. Lu and Y. Li, ACS Appl. Mater. Interfaces, 2014, 6, 11007-11012.

7 E. Mitchell, A. Jimenez, R. K. Gupta, B. K. Gupta, K. Ramasamy, M. Shahabuddin and S. R. Mishra, New J. Chem., 2015, 39, 2181-2187.

8 Y. Zhang, H. Feng, X. Wu, L. Wang, A. Zhang, T. Xia, H. Dong, X. Li and L. Zhang, Int. J. Hydrogen Energy, 2009, 34, 4889-4899.

9 P. Xiao, M. A. Sk, L. Thia, X. Ge, R. J. Lim, J.-Y. Wang, K. H. Lim and X. Wang, Energy Environ. Sci., 2014, 7, 26242629.

10 J. Deng, P. Ren, D. Deng, L. Yu, F. Yang and X. Bao, Energy Environ. Sci., 2014, 7, 1919-1923.

11 M.-R. Gao, J.-X. Liang, Y.-R. Zheng, Y.-F. Xu, J. Jiang, Q. Gao, J. Li and S.-H. Yu, Nat. Commun., 2015, 6, 5982.

12 D. Voiry, H. Yamaguchi, J. Li, R. Silva, D. C. B. Alves, T. Fujita, M. Chen, T. Asefa, V. B. Shenoy, G. Eda and M. Chhowalla, Nat. Mater., 2013, 12, 850-855.

13 L. Cao, S. Yang, W. Gao, Z. Liu, Y. Gong, L. Ma, G. Shi, S. Lei, Y. Zhang, S. Zhang, R. Vajtai and P. M. Ajayan, Small, 2013, 9, 2905-2910.

14 H. Yu, C. Zhu, K. Zhang, Y. Chen, C. Li, P. Gao, P. Yang and Q. Ouyang, J. Mater. Chem. A, 2014, 2, 4551-4557.

15 W. Qiu, J. Xia, S. He, H. Xu, H. Zhong and L. Chen, Electrochim. Acta, 2014, 117, 145-152.

16 X. Wang, J. Ding, S. Yao, X. Wu, Q. Feng, Z. Wang and B. Geng, J. Mater. Chem. A, 2014, 2, 15958-15963.

17 J. M. Soon and K. P. Loh, Electrochem. Solid-State Lett., 2007, 10, A250-A254.

18 B. You and Y. Sun, Adv. Energy Mater., 2016, 6, 1502333.

19 B. Sharma, R. S. Ray and M. Misra, Mater. Lett., 2015, 139, 77-80.

20 Y. Liu, W. Wang, H. Huang, L. Gu, Y. Wang and X. Peng, Chem. Commun., 2014, 50, 4485-4488.

21 S. Ratha and C. S. Rout, ACS Appl. Mater. Interfaces, 2013, 5, 11427-11433.
22 J. Yang, D. Voiry, S. J. Ahn, D. Kang, A. Y. Kim, M. Chhowalla and H. S. Shin, Angew. Chem., Int. Ed., 2013, 52, 1375113754.

23 K.-J. Huang, J.-Z. Zhang, G.-W. Shi and Y.-M. Liu, Mater. Lett., 2014, 131, 45-48.

24 F. Tao, Y.-Q. Zhao, G.-Q. Zhang and H.-L. Li, Electrochem. Commun., 2007, 9, 1282-1287.

25 D. Kong, J. J. Cha, H. Wang, H. R. Lee and Y. Cui, Energy Environ. Sci., 2013, 6, 3553-3558.

26 Y. Sun, C. Liu, D. C. Grauer, J. Yano, J. R. Long, P. Yang and C. J. Chang, J. Am. Chem. Soc., 2013, 135, 17699-17702.

27 M. S. Faber, M. A. Lukowski, Q. Ding, N. S. Kaiser and S. Jin, J. Phys. Chem. C, 2014, 118, 21347-21356.

28 Q. Wang, L. Jiao, H. Du, W. Peng, Y. Han, D. Song, Y. Si, Y. Wang and H. Yuan, J. Mater. Chem., 2011, 21, 327-329.

29 Q. Wang, L. Jiao, H. Du, Y. Si, Y. Wang and H. Yuan, J. Mater. Chem., 2012, 22, 21387-21391.

30 J.-C. Xing, Y.-L. Zhu, Q.-W. Zhou, X.-D. Zheng and Q.-J. Jiao, Electrochim. Acta, 2014, 136, 550-556.

31 P. Justin and G. Ranga Rao, Int. J. Hydrogen Energy, 2010, 35, 9709-9715.

32 R. S. Ray, B. Sarma, A. L. Jurovitzki and M. Misra, Chem. Eng. J., 2015, 260, 671-683.

33 B. You, N. Jiang, M. Sheng and Y. Sun, Chem. Commun., 2015, 51, 4252-4255.

34 L. Zhang, H. B. Wu and X. W. Lou, Chem. Commun., 2012, 48, 6912-6914.

35 W. Li, K. Xu, L. An, F. Jiang, X. Zhou, J. Yang, Z. Chen, R. Zou and J. Hu, J. Mater. Chem. A, 2014, 2, 1443-1447.

36 M. A. Lukowski, A. S. Daniel, F. Meng, A. Forticaux, L. Li and S. Jin, J. Am. Chem. Soc., 2013, 135, 10274-10277.

37 H. Zhang, Y. Li, G. Zhang, P. Wan, T. Xu, X. Wu and X. Sun, Electrochim. Acta, 2014, 148, 170-174.

38 S. Peng, L. Li, X. Han, W. Sun, M. Srinivasan, S. G. Mhaisalkar, F. Cheng, Q. Yan, J. Chen and S. Ramakrishna, Angew. Chem., Int. Ed., 2014, 53, 1259412599.

39 X. Zou and Y. Zhang, Chem. Soc. Rev., 2015, 44, 5148-5180. 